\title{
Skin dissemination of myeloma
}

\author{
Kaja Męcińska-Jundziłł' ${ }^{1}$ Agnieszka Białecka ${ }^{1}$, Urszula Adamska ${ }^{1}$, Adam Cichewicz ${ }^{1}$, Anna Skórczewska ${ }^{2}$, \\ Jacenty Szukalski², Jarosław Czyż ${ }^{3}$, Arkadiusz Jundziłt ${ }^{4}$, Rafał Czajkowski ${ }^{1}$
}

\begin{abstract}
${ }^{1}$ Chair of Dermatology, Sexually Transmitted Diseases and Immunodermatology, Faculty of Medicine in Bydgoszcz, Nicolaus Copernicus University in Torun, Poland

2Department of Clinical Pathomorphology, Collegium Medicum in Bydgoszcz, Nicolaus Copernicus University in Torun, Poland ${ }^{3}$ Department of Hematology, Collegium Medicum in Bydgoszcz, Nicolaus Copernicus University in Torun, Poland

${ }^{4}$ Department of Plastic, Reconstructive and Aesthetic Surgery, Collegium Medicum in Bydgoszcz, Nicolaus Copernicus University in Torun, Poland
\end{abstract}

Adv Dermatol Allergol 2017; XXXIV (6): 622-624

DOI: https://doi.org/10.5114/pdia.2016.62463

According to the WHO classification of tumors of hematopoietic and lymphoid tissues, there are three types of plasma cell neoplasms: plasma cell myeloma (PCM), solitary plasmacytoma of bone and extraosseous plasmacytoma [1]. These tumors are characterized by proliferation of immature or atypical plasma cells in the bone marrow or tissue out of the marrow. We report a case of a patient with cutaneous involvement associated to multiple myeloma (MM), which is rarely seen [2].

A 72-year-old man was admitted to the Department of Dermatology to diagnose firm subcutaneous nodules of violaceous color located at the trunk, abdomen, limbs and scalp (Figure 1). The patient suffered from numerous internal disorders, including immunoglobulin $\mathrm{G} \kappa$ multiple myeloma, type 2 diabetes, hypertension, stable angina pectoris, chronic gastritis, ulcerative colitis, asthma, prostatic hypertrophy and hypercholesterolemia and took insulin therapy, indapamide, acyclovir, ramipril, amlodipine, doxazosin, vinpocetine, ciclesonide, ipratropium bromide and salmeterol. The first symptoms of multiple myeloma occurred 2 years earlier as episodes of anemia and severe pain of the lumbar spine. Bone marrow biopsy showed $42.4 \%$ of plasmocytes and the skull X-ray revealed disseminated osteolytic lesions. Immunofixation confirmed the presence of the kappa type IgG monoclonal protein. The patient was treated with cyclophosphamide, thalidomide and dexamethasone for a year and partial remission was achieved. After 5 months, recurrence of the disease occurred. Treatment with bortezomib, cyclophosphamide and dexamethasone was initiated and continued for 6 months. One month before the completion of therapy, skin lesions occurred as described above. The patient was admitted to the Department of Dermatology. Laboratory examinations showed
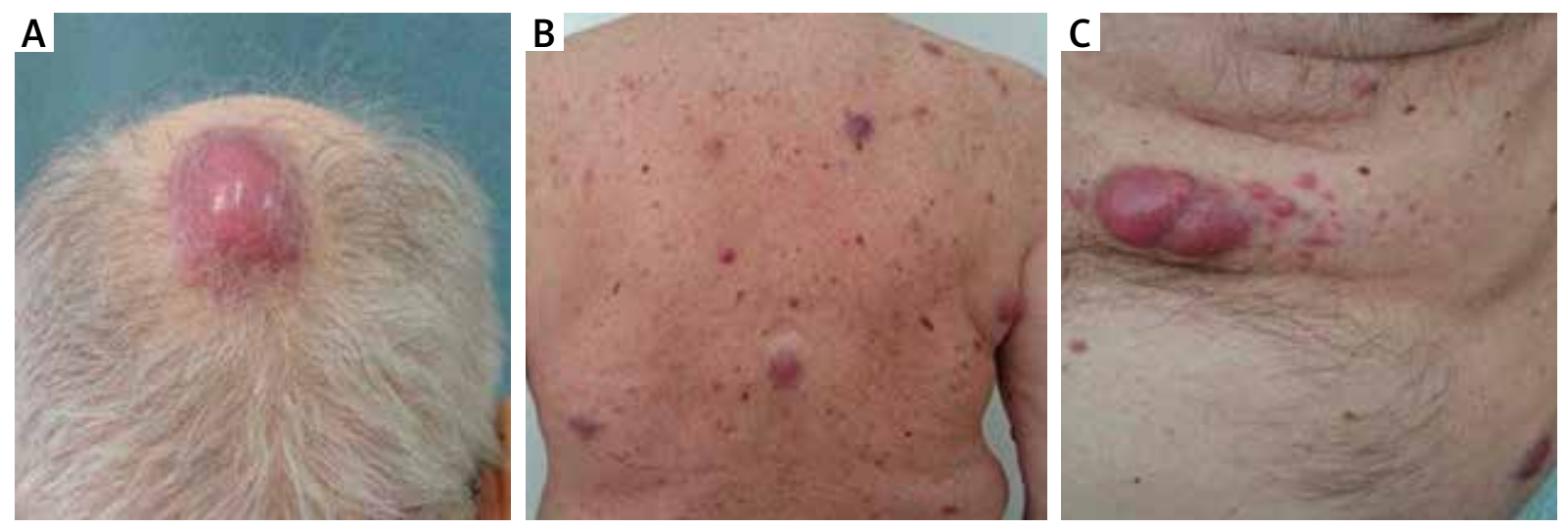

Figure 1. Skin lesions - subcutaneous nodules of violaceous color affecting the trunk, abdomen, limbs and scalp

Address for correspondence: Kaja Męcińska-Jundzitł MD, PhD, Chair of Dermatology, Sexually Transmitted Diseases and Immunodermatology, Collegium Medicum, Nicolaus Copernicus University, 9 Sklodowskiej-Curie St, 85-094 Bydgoszcz, Poland, phone: +48 602404 829, e-mail: kaja_cm@wp.pl

Received: 3.08 .2016 , accepted: 5.09 .2016 . 

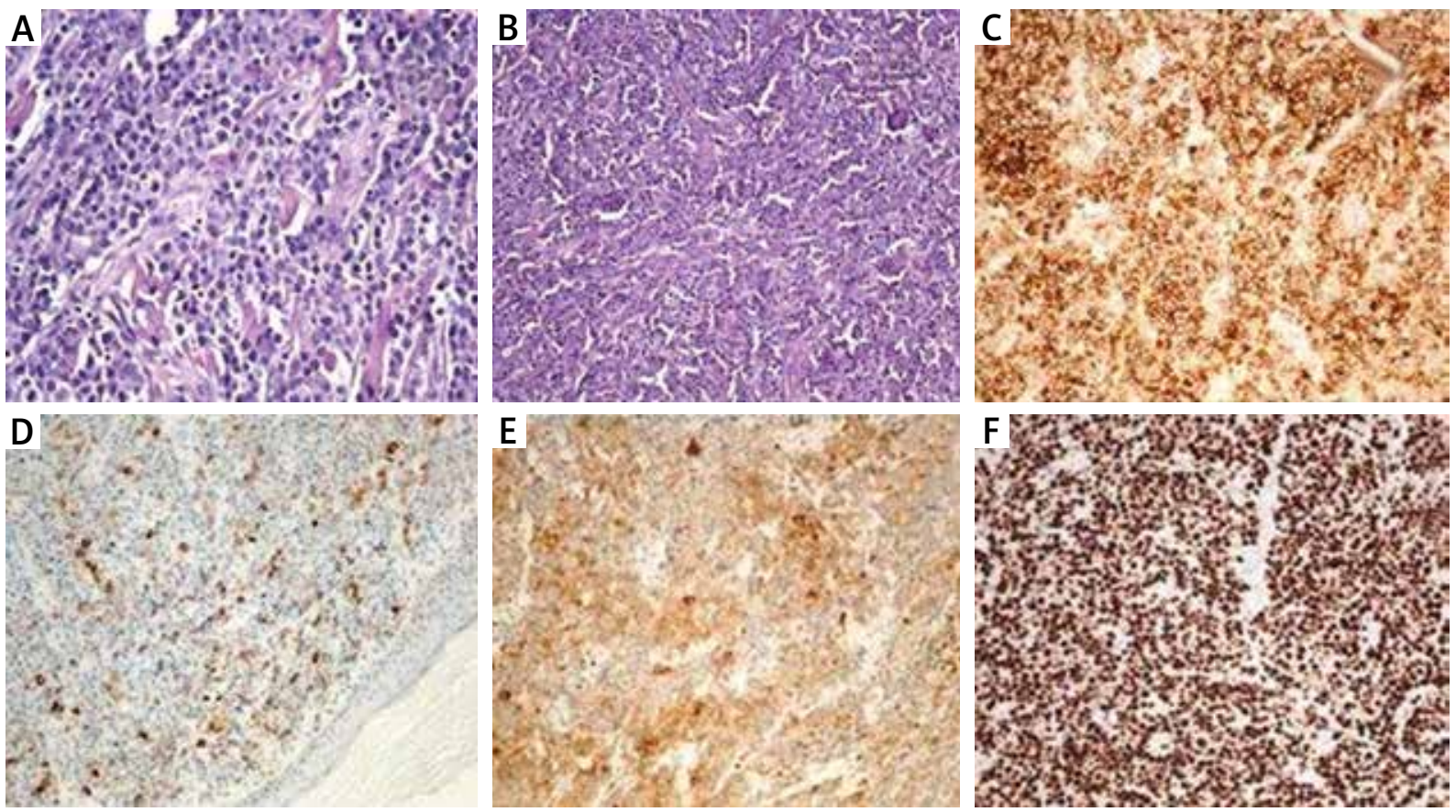

Figure 2. A, B - Histopathology of the nodule - infiltration of plasmacytoma cells. C-F - Immunohistochemistry - plasmablastic lymphoma with round and extended cells with abundant, basophilic cytoplasm, eccentrically localized nuclei containing expressed nucleoli, giant cells and stroma fibrosis. Immunohistochemical characteristics: CD20, CD5, CD10, CD30 - negative reaction; CD138 (C), CD79 $\alpha$ (D), bcl-2 (E) - positive reaction; Ki67 (F) up to $100 \%$ of cells with positive reaction

pancytopenia and an elevated C-reactive protein level. Abdominal ultrasonography showed gallbladder polyps and chest $X$-ray revealed a complete fracture of the sixth left rib. Histopathology of the nodule demonstrated infiltration of plasmacytoma cells (Figures 2 A, B). Immunohistochemistry was characteristic of plasmablastic lymphoma with round and extended cells with abundant, basophilic cytoplasm, eccentrically localized nuclei containing expressed nucleoli, giant cells and stroma fibrosis. Immunohistochemical characteristics: CD20, CD5, CD10, CD30 - negative reaction; CD138, CD79 $\alpha$, bcl-2 - positive reaction; Ki67 up to $100 \%$ of cells with positive reaction (Figures $2 \mathrm{C}-\mathrm{F}$ ). Because of the disease recurrence with numerous skin nodules, the patient was treated with lenalidomide and dexamethasone for 3 months, but the treatment had to be stopped due to pancytopenia. No skin lesions regression was observed during the treatment. The palliative therapy with dexamethasone was initiated. The patient died soon after with the features of active disease.

Approximately 100 cases of specific cutaneous lesions in patients with MM were described. The cutaneous tumors may develop as direct extensions of bone lesions or very rarely independently from bone involvement what we observed in our patient. Skin infiltration of MM usually is an expression of clinical progress and in some cases is the first sign of the disease. Clinically, skin lesions usually appear as multiple papules or cutane- ous and subcutaneous nodules in diameter ranging from $1 \mathrm{~cm}$ to $5 \mathrm{~cm}$. The nodules are firm, have smooth surface and red to violaceous color. Skin lesions can affect any location but most frequently they appear on the trunk and abdomen, next on the scalp, face, neck, lower extremities and upper extremities [3]. Primary cutaneous plasmacytoma and secondary plasmacytoma cannot be differentiated in histopathological examination. Histopathologically, plasma cells infiltrate in nodular or diffuse pattern involving the dermis and less often also the subcutis [4]. Especially, in secondary cutaneous pasmacytoma infiltration can occur in interstitial pattern, where plasma cells are located between the collagen bundles simulating a benign inflammatory infiltrate. The maturity of plasma cells varies. More often they are atypical, have angulated and molded borders, pleomorphic nuclei with large nucleoli and granular eosinophilic cytoplasm. In poorly differentiated lesions plentiful binucleate and multinucleate plasma cells with mitoses are present [5]. Immunohistochemical studies show monoclonal plasma cells with strong immunoexpression of CD79A, CD38, and CD138 [4]. The differential diagnosis includes skin metastases from internal cancers, sarcoidosis, amelanotic melanoma, T-cell lymphoma, Merkel cell carcinoma, tuberculosis, cryptococcosis and cutaneous leishmaniasis. However, medical history of a patient should lead as to direction of plasmacytoma associated with MM. In conclusion, cutaneous involvement in MM is infrequent. 
As shows the case of our patient it is a poor prognostic sign, difficult to treat and patients usually die within 12 months after establishing a diagnosis [2, 3, 6].

\section{Conflict of interest}

The authors declare no conflict of interest.

\section{References}

1. Campo E, Swerdlow SH, Harris NL, et al. The 2008 WHO classification of lymphoid neoplasms and beyond: evolving concepts and practical applications. Blood 2011; 117: 5019-32.

2. Jurczyszyn A, Olszewska-Szopa M, Hungria V, et al. Cutaneous involvement in multiple myeloma: a multi-institutional retrospective study of 53 patients. Leuk Lymphoma 2016; 57: 2071-6.

3. Requena L, Kutzner H, Palmedo G, et al. Cutaneous involvement in multiple myeloma: a clinicopathologic, immunohistochemical, and cytogenetic study of 8 cases. Arch Dermatol 2003; 139: 475-86.

4. Rongioletti F, Patterson JW, Rebora A. The histological and pathogenetic spectrum of cutaneous disease in monoclonal gammopathies. J Cutan Pathol 2008; 38: 705-21.

5. Patterson JW, Parsons JM, White RM, et al. Cutaneous involvement of multiple myeloma and extramedullary plasmacytoma. J Am Acad Dermatol 1988; 19: 879-90.

6. Saback TL, Botelho LFF, Enokihara MM, et al. Multiple primary cutaneous plasmacytoma: first reported case in Brazil. An Bras Dermatol 2012; 87: 629-31. 